\title{
Transmembrane protein with unknown function 16A overexpression promotes glioma formation through the nuclear factor- $\kappa B$ signaling pathway
}

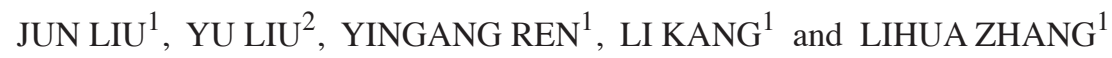 \\ Departments of ${ }^{1}$ Geriatrics and ${ }^{2}$ Neurology, Tangdu Hospital, \\ Fourth Military Medical University, Xi'an, Shaanxi 710038, P.R. China
}

Received July 18, 2013; Accepted January 2, 2014

DOI: $10.3892 / \mathrm{mmr} .2014 .1888$

\begin{abstract}
Ion channels have been suggested to be important in the development and progression of tumors, however, chloride channels have rarely been analyzed in tumorigenesis. More recently, transmembrane protein with unknown function $16 \mathrm{~A}$ (TMEM16A), hypothesized to be a candidate calcium-activated $\mathrm{Cl}^{-}$channel, has been found to be overexpressed in a number of tumor types. Although several studies have implicated the overexpression of TMEM16A in certain tumor types, the exact role of TMEM16A in gliomas and the underlying mechanisms in tumorigenesis, remain poorly understood. In the present study, the role of TMEM16A in gliomas and the potential underlying mechanisms were analyzed. TMEM16A was highly abundant in various grades of gliomas and cultured glioma cells. Knockdown of TMEM16A suppressed cell proliferation, migration and invasion. Furthermore, nuclear factor $-\kappa \mathrm{B}(\mathrm{NF}-\kappa \mathrm{B})$ was activated by overexpression of TMEM16A. In addition, TMEM16A regulated the expression of NF- $\mathrm{BB}-$ mediated genes, including cyclin D1, cyclin E and c-myc, involved in cell proliferation, and matrix metalloproteinases (MMPs)-2 and MMP-9, which are associated with the migration and invasion of glioma cells. Collectively, results of the present study provide evidence for the involvement of TMEM16A in gliomas and the potential mechanism through which TMEM16A promotes glioma formation.
\end{abstract}

Correspondence to: Dr Lihua Zhang, Department of Geriatrics, Tangdu Hospital, Fourth Military Medical University, 1 Xinsi Road, Baqiao, Xi'an, Shanxi 710038, P.R. China

E-mail: lihuazhangen@163.com

Abbreviations: CaCCs, calcium-activated chloride channels; TMEM16A, transmembrane protein with unknown function 16A; MMP, matrix metalloproteinase; NF- $\kappa B$, nuclear factor- $\kappa B$; CFTR, cystic fibrosis transmembrane conductance regulator; BrdU, 5-bromo-2-deoxyuridine

Key words: calcium-activated chloride channels, nuclear factor- $\kappa \mathrm{B}$, glioma, transmembrane protein with unknown function 16A

\section{Introduction}

In previous years, the association between ion channels and tumors has drawn particular attention. Increasing evidence has demonstrated that ion channels are involved in the regulation of tumor progression, including potassium (1-3), calcium (4) and sodium channels $(5,6)$. Therefore, understanding the underlying molecular mechanisms of ion channels in tumorigenesis, and tumor progression and migration provides novel insights into tumor pathogenesis, and also identifies potential targets for tumor prevention and treatment.

Chloride channels are expressed ubiquitously and are important in various cellular processes, including the cell cycle and proliferation, cell volume modulation, epithelial secretion and membrane excitability (7). To date, chloride channel family members, including CLC proteins, ionotropic receptors for $\gamma$-aminobutyric acid and glycine, and the cystic fibrosis transmembrane conductance regulator (CFTR), have been described (8). Calcium-activated chloride channels (CaCCs) are reported to be major modulators of cell volume and epithelial secretion (9). However, the composition of $\mathrm{CaCC}$ remains unknown. More recently, transmembrane protein with unknown function 16 (TMEM16) A, also known as anoctamin 1, has been hypothesized to be a candidate CaCC (10-12). TMEM16A belongs to the TMEM16 family, which consists of nine other members, including TMEM16B-K, characterized by eight transmembrane segments and a highly conserved domain of unknown function. TMEM16A has been demonstrated to be involved in the regulation of gastrointestinal tract motility, epithelial fluid transport and saliva production (13-16). CaCCs have been found to be proapoptotic, acting as tumor suppressor genes in mammary epithelium (17). However, bestrophin, a putative $\mathrm{CaCC}$ that promotes cell proliferation, has been shown to be overexpressed in colon cancer (18). Notably, TMEM16A is localized on chromosome 11q13, a region that is frequently amplified in human cancers, including those of the head and neck, esophagus, bladder and breast (19-21). Therefore, TMEM16A dysregulation may be associated with tumor progression. As hypothesized, several studies have revealed that TMEM16A is overexpressed in various tumor types, including gastrointestinal stromal tumors, esophageal cancers, and head and neck cancers (22-24). However, the 
molecular mechanisms of TMEM16A in the regulation of tumors remains poorly understood.

Several studies have hypothesized that chloride channels are associated with the nuclear factor $-\kappa \mathrm{B}(\mathrm{NF}-\kappa \mathrm{B})$ signaling pathway (25-29). NF- $\kappa \mathrm{B}$, which is tightly controlled, regulates a wide range of cellular processes and is extensively involved in cancer progression (30). Normally, the binding of $N F-\kappa B$ to inhibitory inhibitor of $\kappa \mathrm{B}(\mathrm{I} \kappa \mathrm{B})$ proteins inactivates $\mathrm{NF}-\kappa \mathrm{B}$ in the cytoplasm (31). Degradation of $\mathrm{I} \kappa \mathrm{B}$ proteins releases $\mathrm{NF}-\kappa \mathrm{B}$ and enables it to translocate to the nucleus and activate target genes. Dysregulation of this process is involved in a number of diseases $(32,33)$. NF- $\kappa \mathrm{B}$ has also been demonstrated to be a critical regulator of tumorigenesis involved in cell survival, metastasis and angiogenesis (31). However, although TMEM16A has been hypothesized to be a transmembrane protein regulating cellular ion exchanges, its role in the regulation of the NF- $\mathrm{NB}$ signal pathway remains unclear.

To date, no studies have determined the exact role of TMEM16A in gliomas. The aim of the present study was to determine whether TMEM16A is involved in gliomas and the potential underlying mechanisms.

\section{Materials and methods}

Cell lines and cell culture. One normal human astrocyte line (SVGp12) and four glioma cell lines (U87MG, U118, U251 and SHG44) were obtained from the American Type Culture Collection (Manassas, VA, USA). All cells were maintained according to standard protocols. Briefly, cells were cultured in Dulbecco's modified Eagle's medium (DMEM) supplemented with $10 \%$ fetal bovine serum to which $100 \mathrm{U} / \mathrm{ml}$ penicillin, $100 \mu \mathrm{g} / \mathrm{ml}$ streptomycin and $2 \mathrm{mM}$ L-glutamate were added. All cells were cultured at $37^{\circ} \mathrm{C}$ with $5 \% \mathrm{CO}_{2}$ in an incubator (Invitrogen Life Technologies, Carlsbad, CA, USA).

Tumor tissue preparation. All tumor tissues were obtained from Tangdu Hospital (Xi'an, China) according to institutional guidelines for consent with the approval of patients and the hospital. Brain tissue was obtained from 20 patients with gliomas (eight females and 12 males; median age, 43.6 years; range, 16-78 years; five each of grade I, II, III and IV). Normal brain tissue samples were obtained from five patients with brain trauma (median age, 42 years; range, 21-68 years). All samples were obtained from the initial surgery, prior to chemotherapy and radiotherapy. The malignant grades of the tumor tissues were analyzed as per the standard classification established by the World Health Organization (34).

Quantitative polymerase chain reaction ( $q P C R$ ) analysis. Total RNA was extracted from cultured cells using TRIzol reagent (Invitrogen Life Technologies), following the manufacturer's instructions. In total, $\leq 5 \mu \mathrm{g}$ RNA was reverse-transcribed into cDNA using Maloney-murine leukemia virus reverse transcriptase (Clontech Laboratories, Inc., Palo Alto, CA, USA). The cDNA molecules were used as templates for qPCR. Genes were amplified using primers for TMEM16A, cyclin D1, cyclin E and c-myc. The primers were as follows: TMEM16A forward, 5'-CACAAGAGAGCCTCGGGTAG-3' and reverse, 5'-ATCTTCACAAACCCGACACC-3'; cyclin D1 forward, 5'-GCCAACCTCCTCAACGACCGG-3' and reverse, 5'-GTCCATGTTCTGCTGGGCCTG-3'; cyclin E forward, 5'-GTCCTGGCTGAATGATACATC-3' and reverse, 5'-CCCTATTTTGTTCAGACAACATGGC-3'; c-myc forward 5'-ACACATCAGCACAACTACGC-3' and reverse, 5'-CCTCTTGACATTCTCCTCGGT-3'; GAPDH forward, 5'-CGGAGTCAACGGATTTGGTCGTAT-3' and reverse 5'-AGCCTTCTCCATGGTGGTGAA-3'. The qPCR

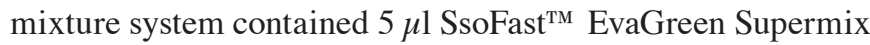
(Bio-Rad, Hercules, CA, USA), $1 \mu \mathrm{l}$ cDNA (diluted at 1:50) and $2 \mu \mathrm{l}$ each of the forward and reverse primers $(1 \mu \mathrm{M})$, to a final volume of $10 \mu \mathrm{l}$. The PCR procedure was as follows: $94^{\circ} \mathrm{C}$ for $4 \mathrm{~min} ; 94^{\circ} \mathrm{C}$ for $20 \mathrm{sec}, 55^{\circ} \mathrm{C}$ for $30 \mathrm{sec}$ and $72^{\circ} \mathrm{C}$ for $20 \mathrm{sec}$; $2 \mathrm{sec}$ for plate reading for 35 cycles; melting curve, $65-95^{\circ} \mathrm{C}$. GAPDH was used as a control for normalizing the gene expression. Three independent experiments were performed. The data obtained were analyzed by the $2^{-\Delta \Delta \mathrm{Ct}}$ method and statistically analyzed as described previously (35), followed by Student's unpaired sample t-test.

Nuclear protein extraction. Nuclear proteins were extracted using a Nuclear Extraction kit (Sangon Biotech, Shanghai, China) according to the manufacturer's instructions. Briefly, cells were lysed in cytoplasmic buffer containing protease inhibitors, mixed and incubated for $15 \mathrm{~min}$ at $4^{\circ} \mathrm{C}$. Next, cells were centrifuged at $12,000 \mathrm{rpm}$ for $20 \mathrm{~min}$ at $4^{\circ} \mathrm{C}$. Supernatants containing cytoplasmic extraction products were collected. Cell sediments were collected and resuspended in nucleus buffer for $10 \mathrm{~min}$ at $4^{\circ} \mathrm{C}$. Next, the sample was centrifuged at $12,000 \mathrm{rpm}$ for $10 \mathrm{~min}$ at $4^{\circ} \mathrm{C}$ and the supernatant was collected for analysis.

Western blotting. A total of $20-30 \mu \mathrm{g}$ protein was fractionated by $12 \%$ SDS-PAGE electrophoresis and transferred to nitrocellulose membranes (Amersham, Little Chalfont, UK). The membrane was treated under agitation and blocking at room temperature, with $2 \%$ non-fat dry milk in Tris-buffered saline (TBS) for $1 \mathrm{~h}$, followed by incubation with primary rabbit anti-human TMEM16A, IкB, p65, MMP-2, MMP-9, GAPDH and Histone polyclonal antibodies. (Santa Cruz Biotechnology, CA, USA). Antibodies were diluted in blocking buffer $(1: 2,000)$ and incubated with the membrane at $4^{\circ} \mathrm{C}$ overnight. Next, membranes were washed three times with TBS and Tween 20 [TBST; $10 \mathrm{mM}$ Tris- $\mathrm{HCl}$ (pH 7.5), $150 \mathrm{mM} \mathrm{NaCl}$ and $0.05 \%$ Tween-20] for $10 \mathrm{~min}$ at room temperature. Subsequently, the membrane was incubated in horseradish peroxidase (HRP)-conjugated secondary antibody goat anti-rabbit Ig G (diluted 1:5,000 in the blocking buffer; Boster Biological Technology Ltd., Wuhan, China) for $1 \mathrm{~h}$. Following washing three times with TBST and once with TBS each for $10 \mathrm{~min}, 1 \mathrm{ml}$ 4-chloro-1-naphthol was used as the HRP substrate, with $9 \mathrm{ml} \mathrm{TBS}$ and $6 \mu \mathrm{l} \mathrm{H}_{2} \mathrm{O}_{2}$, to visualize the target protein in the dark for 5-30 $\mathrm{min}$.

Cell transfection and luciferase assay. The full length cDNA-encoding sequence of TMEM16A was subcloned into pcDNA3.1 vectors according to standard protocols. Small interfering RNAs (siRNAs) targeting TMEM16A and control siRNA were purchased from Santa Cruz Biotechnology. Cells were transfected with vectors or siRNA according to the manufacturer's instructions. Briefly, plasmid DNA $(1 \mu \mathrm{g})$ or siRNA (30 pmol) were diluted in $500 \mu \mathrm{l}$ DMEM with $5 \mu \mathrm{l}$ Lipofectamine (Invitrogen Life Technologies), mixed and 
incubated at room temperature for $15 \mathrm{~min}$. Next, the mixtures were added to the cells at a final volume of $3 \mathrm{ml}$ medium and incubated for $24 \mathrm{~h}$. Cells were co-transfected with the promoter luciferase reporter plasmid and harvested after $24 \mathrm{~h}$. Luciferase activity was analyzed by the Dual Luciferase Reporter Assay system (Promega Corporation, Madison, WA, USA).

Cell proliferation assay. For the MTT assay, cells were plated in 96-well plates and cultured under standard conditions until they reached $80 \%$ confluency. Plasmids or siRNA were transfected, according to standard protocols, and incubated with cells for the indicated times. Next, the initial culture medium was replaced with fresh medium containing MTT $(5 \mathrm{mg} / \mathrm{ml}$ in PBS; $200 \mu \mathrm{l} /$ well; Sigma, St. Louis, MO, USA) and incubated for an additional $4 \mathrm{~h}$. The formazan was dissolved in dimethylsulfoxide (150 $\mu \mathrm{l} /$ well; Sigma) for $10 \mathrm{~min}$ and the absorbance at $490 \mathrm{~nm}$ was determined with an ELISA reader (Bio-Tek Instruments, Winooski, VA, USA). Each cell viability assay was performed in quadruplicate and repeated three times. For the 5-bromo-2-deoxyuridine (BrdU) assay, a BrdU Cell Proliferation Assay kit (Millipore, Billerica, MA, USA) was used. A total of $10 \mu \mathrm{l}$ BrdU solution was added per well and incubated with cells for $2 \mathrm{~h}$. Next, the medium was discarded and $100 \mu \mathrm{l} /$ well fixing/denaturing solution was added for $15 \mathrm{~min}$. Following this, the solution was removed and $100 \mu \mathrm{l} /$ well antibody detection solution was added and incubated for $1 \mathrm{~h}$ at room temperature. Thereafter, plates were washed three times with wash buffer, followed by the addition of $100 \mu \mathrm{l} /$ well prepared HRP-conjugated secondary antibody solution, and incubated for $30 \mathrm{~min}$ at room temperature. Plates were washed three times with wash buffer and $100 \mu 1$ 3,3',5,5'-tetramethylbenzidine substrate was added prior to incubation for $30 \mathrm{~min}$ at room temperature. The volume of BrdU incorporated into the cells was determined at $450 \mathrm{~nm}$ by a microplate reader (Bio-Rad). Data are expressed as the mean \pm standard error of the mean and differences were analyzed by Student's-t test.

Cell invasion and migration assays. Cells were suspended in a volume of $50 \mu \mathrm{l}$ serum-free medium, which was then added to the upper chamber of chemotaxis chambers (Neuro Probe, Inc., Gaithersburg, MD, USA). Complete medium was added to the lower chamber. Polycarbonate membrane was placed between the two chambers and culture medium, supplemented with $20 \mu 1$ Matrigel (BD Biosciences, Franklin Lakes, NJ, USA), was applied. Cells were incubated for $36 \mathrm{~h}$ at $37^{\circ} \mathrm{C}$. Next, the membrane was fixed and stained with methanol and Giemsa, respectively. In total, $\sim 10$ visual fields were randomly selected and quantified per membrane. Each sample was assayed in triplicate. A similar system was performed for the migration assay.

Statistical analysis. All experiments were performed independently at least three times. Differences between groups were analyzed by Student's t-test and $\mathrm{P}<0.01$ was considered to indicate a statistically significant difference.

\section{Results}

TMEM16A expression is high in glioma tissue. To determine whether TMEM16A is involved in glioma formation, the expression profiles of TMEM16A in the tissue sample

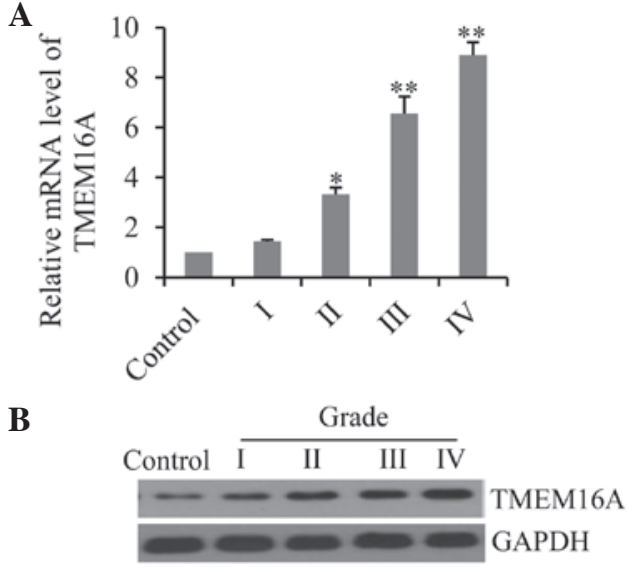

Figure 1. Expression profiles of TMEM16A in gliomas. Analysis of TMEM16A (A) mRNA and (B) protein levels in gliomas of pathological grades I-IV by quantitative polymerase chain reaction and western blotting. GADPH was used as the control. ${ }^{*} \mathrm{P}<0.05$ and ${ }^{* *} \mathrm{P}<0.01$, vs. the control group. TMEM16A, transmembrane protein with unknown function 16A.

A

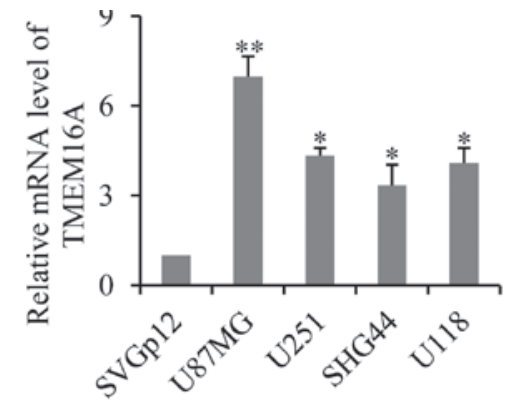

B

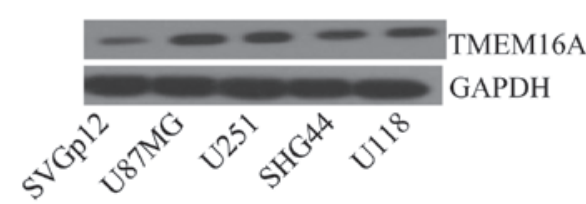

Figure 2. Expression profiles of TMEM16A in various cell lines. (A) mRNA and (B) protein levels were determined in the normal astrocyte line, SVGp12, and glioma cell lines, U87MG, U251, SHG44 and U118, by quantitative polymerase chain reaction and western blotting. GADPH was used as the control. ${ }^{*} \mathrm{P}<0.05$ and ${ }^{* *} \mathrm{P}<0.01$, vs. the control group. TMEM16A, transmembrane protein with unknown function $16 \mathrm{~A}$.

of patients with various pathological grade gliomas were analyzed by qPCR and western blotting. Results demonstrated that mRNA expression levels increased with increasing pathological glioma grades (Fig. 1A), with particularly high levels seen in grade III and IV gliomas. The data were further confirmed by western blotting, which demonstrated that TMEM16A proteins were also overexpressed in glioma tissues and correlated with high pathological grade (Fig. 1B).

TMEM16A expression is high in glioma cell lines. Next, the expression profiles of TMEM16A were measured in four cultured glioma cell lines and one normal astrocyte line. Consistent with results from glioma tissues, TMEM16A demonstrated high mRNA expression levels in the four cultured glioma cell lines, U87MG, U251, SHG44 and U118, compared with the normal astrocyte cell line, SVGp12 (Fig. 2A; $\mathrm{P}<0.05$ ). 
A

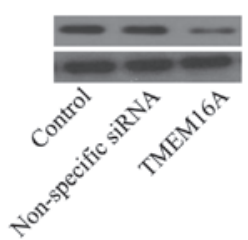

B

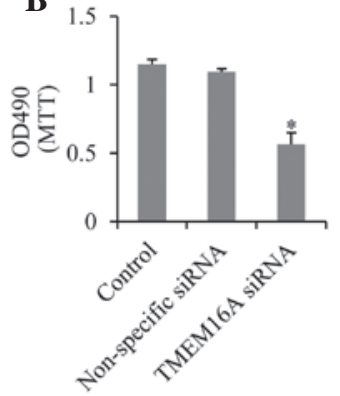

C

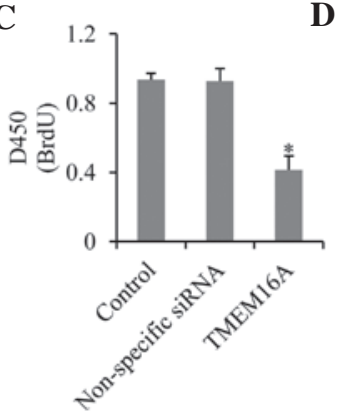

D

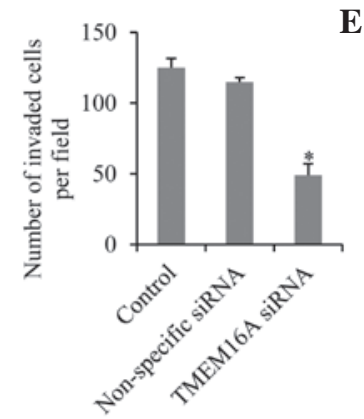

E

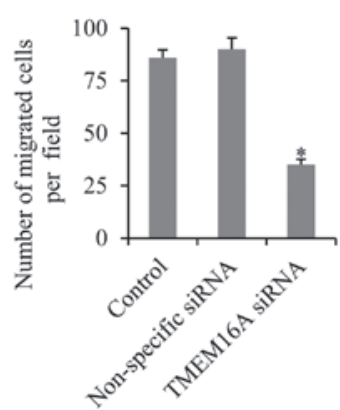

Figure 3. Effect of TMEM16A on cell proliferation, migration and invasion. (A) Western blotting was performed to measure TMEM16A expression in various treatment groups of U87MG cells. Cells were harvested after 72-h transfection. Cell proliferation was analyzed by (B) MTT and (C) BrdU assays. Each cell proliferation assay was performed in quadruplicate and repeated three times. (D) Migration and (E) invasion assays in various treatment groups of U87MG cells. Data are expressed as the mean \pm standard error of the mean and differences were analyzed by Student's-t test. Means were obtained from three individual experiments. "P<0.05, vs. the control group. Control, cells without treatment; nonspecific siRNA, cells transfected with nonspecific siRNA; TMEM16A siRNA, cells transfected with specific siRNA targeting for TMEM16A; siRNA, short interfering RNA; TMEM16A, transmembrane protein with unknown function 16A; OD, optical denisty; BrdU, 5-bromo-2-deoxyuridine.

A

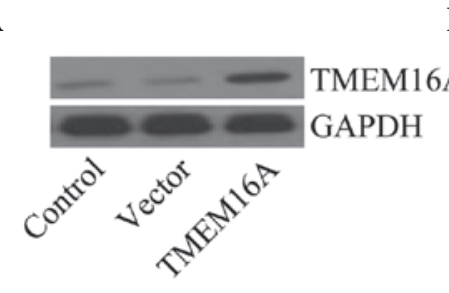

B

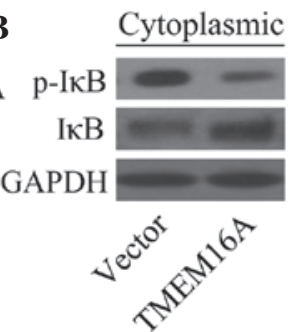

C

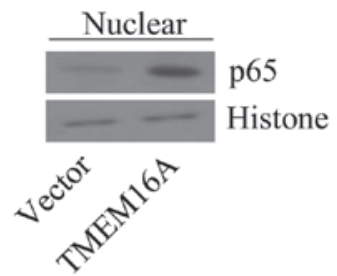

Figure 4. Effect of TMEM16A on the activation of signaling pathways. (A) Western blotting was performed to analyze TMEM16A expression levels in transfected SVGp1 2 cells. Western blotting of key components of NF- $\mathrm{BB}$ in the (B) cytoplasm and (C) nucleus with indicated antibodies. Control, cells without treatment; vector, cells transfected with null vectors; TMEM16A, cells transfected with TMEM16A overexpression vectors; TMEM16A, transmembrane protein with unknown function $16 \mathrm{~A}$; I $\mathrm{B}$, inhibitor of $\kappa \mathrm{B}$; NF- $\kappa \mathrm{B}$, nuclear factor- $\kappa \mathrm{B}$.

Additionally, TMEM16A levels were more abundant in U87MG cells $(\mathrm{P}<0.01)$. These results were further confirmed by western blotting of the protein levels of TMEM16A in these cell lines (Fig. 2B). Collectively, these results indicate that dysregulation of TMEM16A is associated with gliomas.

Alteration of TMEM16A expression impairs cell proliferation, migration and invasion. To investigate the role of TMEM16A in gliomas, the expression of TMEM16A was silenced in U87MG cells by specific siRNAs (Fig. 3A). First, the effects of TMEM16A knockdown on cell proliferation were detected. The MTT assay revealed that cell growth and viability were significantly inhibited compared with controls (Fig. 3B). This was further confirmed by BrdU assay, which demonstrated that cell proliferation was markedly decreased upon knockdown of TMEM16A (Fig. 3C). Furthermore, the migration (Fig.3D) and invasion (Fig. 3E) ability were also significantly impaired upon TMEM16A knockdown. These results indicate that TMEM16A is important in cell proliferation and in the migration and invasion of glioma cells. By contrast, SVGp12 cells transfected with TMEM16A overexpression vectors exhibited high levels of cell proliferation, migration and invasion (data not shown).

TMEM16A is involved in activation of $N F-\kappa B$. Various studies have demonstrated that chloride channels are involved in the activation of $\mathrm{NF}-\kappa \mathrm{B}(25,26)$. In order to assess whether TMEM16A is associated with the activation of NF- $\kappa \mathrm{B}, \mathrm{SVGp12}$ cells were used to generate stable TMEM16A overexpression cell lines. Cells transfected with TMEM16A overexpression vectors exhibited an increase in TMEM16A expression levels compared with the vector control (Fig. 4A). Cytoplasmic and nuclear extracts were prepared from various cell groups. Results revealed that $\mathrm{I} \kappa \mathrm{B} \alpha$ phosphorylation was decreased in TMEM16A overexpressing cells, while unphosphorylated I $\kappa \mathrm{B} \alpha$ was increased in the cytoplasm (Fig. 4B), compared with control vector-transfected cells. Furthermore, the NF- $\kappa$ B subunit p65 accumulated in the nucleus in TMEM16A-overexpressing cells (Fig. 4C). The data suggest that TMEM16A is associated with activation of the NF- $\mathrm{B}$ signaling pathway.

TMEM16A regulates $N F-\kappa B$-mediated gene transcription. In order to further determine whether TMEM16A regulates $\mathrm{NF}-\kappa \mathrm{B}-$ mediated gene transcription in glioma cells, a luciferase reporter vector containing the $\mathrm{NF}-\kappa \mathrm{B}$ response element promoter and TMEM16A siRNA were co-transfected into U87MG cells. Results demonstrated that the luciferase activity was decreased three-fold in TMEM16A-silenced cells compared with the control group (Fig. 5A). In TMEM16A-overexpressing SVGp12 cells, luciferase activity increased four-fold (Fig. 5B). The results indicate that TMEM16A promotes NF- $\kappa \mathrm{B}$-mediated gene transcription.

TMEM16A increases cell proliferation through cyclin D1, cyclin $E$ and $c$-myc activated by $N F-\kappa B$. Various studies have 
A
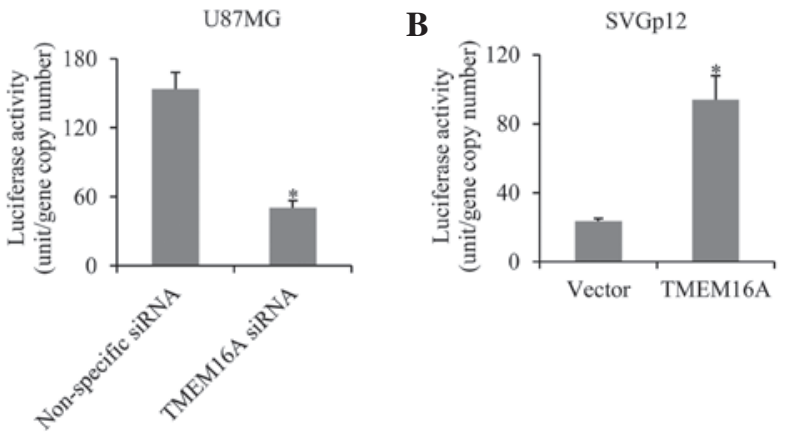

Figure 5. Effect of TMEM16A on NF- $\mathrm{B}$-mediated gene transcription. Detection of luciferase activity in (A) a luciferase reporter vector containing the NF- $\kappa \mathrm{B}$ response element promoter and siRNA co-transfected U87MG cells, and (B) a luciferase reporter vector and overexpression vectors co-transfected with SVGp12 cells. ${ }^{*} \mathrm{P}<0.05$, vs. the non-specific siRNA and vector groups. siRNA, small interfering RNA; TMEM16A, transmembrane protein with unknown function $16 \mathrm{~A} ; \mathrm{NF}-\kappa \mathrm{B}$, nuclear factor- $\kappa \mathrm{B}$.

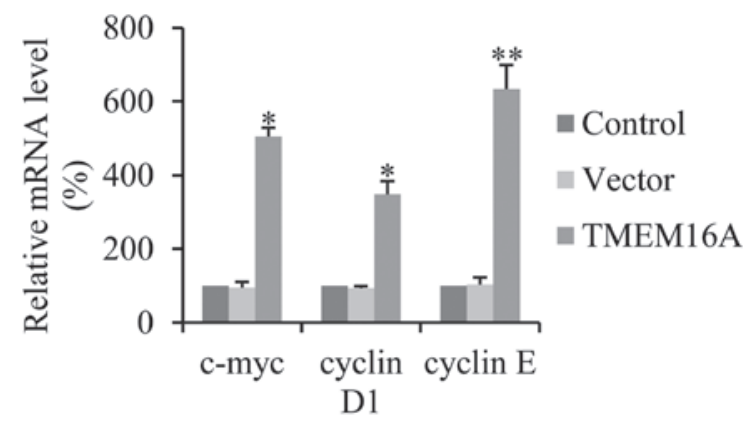

Figure 6. Effect of TMEM16A on nuclear factor- $\kappa$ B-target genes involved in cell proliferation. The transcription levels of c-myc, cyclin D1 and cyclin E were evaluated by quantitative polymerase chain reaction. GADPH was used as a loading control. All experiments were independently performed at least three times. The data are expressed as the mean \pm standard error of the mean and analyzed using Student's t test. ${ }^{*} \mathrm{P}<0.05$ and ${ }^{* *} \mathrm{P}<0.01$, vs. the control group. TMEM16A, transmembrane protein with unknown function 16A.
A

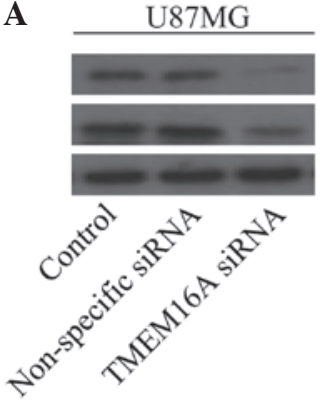

B

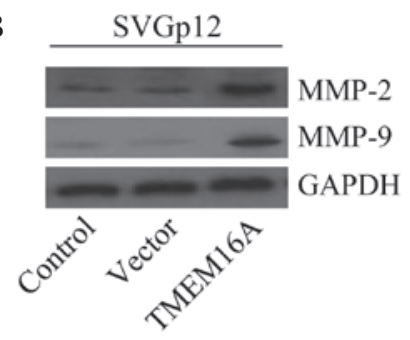

Figure 7. Effect of TMEM16A on MMP expression. Western blotting of MMP-2 and -9 expression in (A) TMEM16A siRNA-transfected U87MG cells and (B) TMEM16A overexpression vector-transfected SVGp12 cells with the indicated antibodies. siRNA, small interfering RNA; MMP, matrix metalloproteinase; TMEM16A, transmembrane protein with unknown function 16A.

demonstrated that $\mathrm{NF}-\kappa \mathrm{B}$ is involved in the regulation of cell proliferation through cyclin D1, cyclin E and c-myc (36-38). In order to define whether overexpression of TMEM16A activates $\mathrm{NF}-\kappa \mathrm{B}-$ mediated cell proliferation, the transcription levels of cyclin D1, cyclin E and c-myc were determined by
qPCR. TMEM16A overexpression, compared with controls, significantly increased the expression of cyclin D1, cyclin E and c-myc in SVGp12 cells (Fig. 6). These results indicate that TMEM16A promotes cell proliferation in glioma cells via the $\mathrm{NF}-\kappa \mathrm{B}$-target gene.

TMEM16A promotes cell migration and invasion by MMP-2 and -9 mediated by $N F-\kappa B$. Previous studies have demonstrated that MMPs, particularly MMP-2 and -9 , are markedly associated with cell migration and invasion of gliomas and are the target genes of $\mathrm{NF}-\kappa \mathrm{B}(39-41)$. The present study aimed to determine whether TMEM16A impaired cell migration and invasion by MMP-2 and -9 , mediated by NF- $\kappa$ B. Results revealed that knockdown of TMEM16A in U87MG cells effectively downregulated MMP-2 and -9 protein expression levels (Fig. 7A). Additionally, it was found that overexpression of TMEM16A in SVGp12 cells markedly upregulated protein expression levels of MMP-2 and -9 (Fig. 7B). These results indicate that TMEM16A is involved in the regulation of MMP-2 and -9 .

\section{Discussion}

In previous years, increasing evidence has demonstrated that chloride channels regulate tumor growth and progression $(42,43)$. In addition, contradictory functions of CaCCs, including upregulation and downregulation of tumor growth, have been reported $(17,18)$. High levels of TMEM16A have been reported in gastrointestinal stromal tumors, squamous cell carcinomas of the head and neck, and esophageal cancer (22-24). In the present study, TMEM16A was demonstrated to be overexpressed in gliomas. Notably, a higher abundance was found in grade III and IV gliomas, indicating that high TMEM16A expression may be correlate with pathological grade. However, low levels of TMEM16A were also detected in normal brain tissues and normal human astrocytes, indicating that TMEM16A may be essential for maintaining basic cellular activities. In addition, TMEM16A has been shown to be expressed in a broad spectrum of mouse and human tissues (44). However, the precise mechanism by which TMEM16A regulates tumor development and progression remains poorly understood.

Chloride channels have previously been hypothesized to be involved in the regulation of cellular proliferation. The present study found that knockdown of TMEM16A significantly suppressed the proliferation of glioma cells. In addition, silencing of TMEM16A downregulated the migration and invasion of glioma cells. It has been reported that TMEM16A overexpression contributes to tumor metastasis by promoting cell motility (45). Recently, TMEM16A was suggested to influence cellular proliferation through activation of the Ras-Raf-mitogen-activated protein kinase-extracellular signal-related kinase 1/2 pathway (46). Blockade of TMEM16A has also been shown to markedly increase the expression of insulin-like growth factor-binding protein 5, a potent anti-angiogenic factor, therefore implicating TMEM16A in the modulation of the tumor microenvironment through the signaling pathway (47). However, it was also demonstrated that suppression of TMEM16A did not affect cell growth in vitro but significantly delayed the growth of xenografts in vivo. 
Mazzone et al (48) reported that inhibition of TMEM16A, by its selective inhibitor T16A(inh)-A01, reduced the proliferation of cultured pancreatic cancer cells and interstitial cells. Liu et al (49) provided data demonstrating that TMEM16A was involved in tumorigenesis and the development of metastatic prostate cancer. Increasing evidence has indicated that TMEM16A is involved in the development and progression of tumors. Thus, further study into the exact role of TMEM16A in tumors is of extreme importance.

Chloride channels have been hypothesized to be involved in the regulation of $\mathrm{NF}-\kappa \mathrm{B}$. A decrease in intracellular chloride concentration activates the $\mathrm{NF}-\kappa \mathrm{B}$ signaling pathway through the chloride channel CLC-3 (25). Inhibition of the CFTR impairs tight junctions through activation of the $\mathrm{NF}-\kappa \mathrm{B}$ signaling pathway (26). However, whether TMEM16A is associated with the activation of $\mathrm{NF}-\kappa \mathrm{B}$ remains unclear. $\mathrm{NF}-\kappa \mathrm{B}$ is important in the regulation of various cellular functions that are frequently overactivated in tumor cells. In order to determine whether TMEM16A overexpression is involved in the activation of $\mathrm{NF}-\kappa \mathrm{B}$, in the present study, the key components of the activation of $N F-\kappa B$ were examined in TMEM16A-expressing SVGp12 cells. Phosphorylation levels of the inhibitor of $\mathrm{NF}-\kappa \mathrm{B}, \mathrm{I} \kappa \mathrm{B} \alpha$, were high following TMEM16A overexpression, which was further confirmed by the accumulation of the $\mathrm{NF}-\kappa \mathrm{B}$ subunit $\mathrm{p} 65$ protein in the nucleus. Furthermore, a luciferase reporter assay also demonstrated that overexpression of TMEM16A significantly upregulated $\mathrm{NF}-\kappa \mathrm{B}-$ mediated gene transcription activity. These results suggest that the increased activity of $N F-\kappa B$ is associated with TMEM16A overexpression. Thus, the present study may present a mechanism for the role of TMEM16A in tumors.

$\mathrm{NF}-\kappa \mathrm{B}$ has been implicated in the regulation of glioma cell proliferation through the upregulation of oncogenes, including cyclin D1, cyclin E, and c-myc (50-54). The present study revealed that overexpression of TMEM16A significantly increased transcription levels of these genes, which may explain why TMEM16A promotes cell proliferation in gliomas. MMPs, the target genes of $\mathrm{NF}-\kappa \mathrm{B}$, have long been implicated in tumor progression processes, for example cell migration and invasion (39). In particular, MMP-2 and -9 are heavily implicated in tumor progression and have been shown to regulate cell migration and the invasion of gliomas $(40,41)$. In the present study, TMEM16A was demonstrated to be closely associated with the expression of MMP-2 and -9.

The present study has shown that TMEM16A is overexpressed in gliomas, particularly in high grade gliomas (III and IV). Furthermore, it has been demonstrated that TMEM16A overexpression promotes cell proliferation, migration and invasion through activation of the $\mathrm{NF}-\kappa \mathrm{B}$ signaling pathway. In conclusion, the present study provides novel insights into the role of TMEM16A in the development and progression of gliomas, indicating that this protein may act as a potential therapeutic target. However, the specific role of TMEM16A in the regulation of tumors requires further study.

\section{References}

1. Stühmer W, Alves F, Hartung F, Zientkowska M and Pardo LA: Potassium channels as tumour markers. FEBS Lett 580: 2850-2852, 2006.
2. Lastraioli E, Taddei A, Messerini L, et al: hERG1 channels in human esophagus: evidence for their aberrant expression in the malignant progression of Barrett's esophagus. J Cell Physiol 209: 398-404, 2006

3. Masi A, Becchetti A, Restano-Cassulini R, et al: hERG1 channels are overexpressed in glioblastoma multiforme and modulate VEGF secretion in glioblastoma cell lines. Br J Cancer 93: 781-792, 2005.

4. Panner A and Wurster RD: T-type calcium channels and tumor proliferation. Cell Calcium 40: 253-259, 2006.

5. Diss JK, Stewart D, Pani F, et al: A potential novel marker for human prostate cancer: voltage-gated sodium channel expression in vivo. Prostate Cancer Prostatic Dis 8: 266-273, 2005.

6. Fraser SP, Diss JK, Chioni AM, et al: Voltage-gated sodium channel expression and potentiation of human breast cancer metastasis. Clin Cancer Res 11: 5381-5389, 2005.

7. Furst J, Gschwentner M, Ritter M, et al: Molecular and functional aspects of anionic channels activated during regulatory volume decrease in mammalian cells. Pflugers Arch 444: 1-25, 2002.

8. Ferrera L, Caputo A and Galietta LJ: TMEM16A protein: a new identity for $\mathrm{Ca}(2+)$-dependent $\mathrm{Cl}^{-}$channels. Physiology (Bethesda) 25: 357-363, 2010

9. Hartzell C, Putzier I and Arreola J: Calcium-activated chloride channels. Annu Rev Physiol 67: 719-758, 2005.

10. Caputo A, Caci E, Ferrera L, et al: TMEM16A, a membrane protein associated with calcium-dependent chloride channel activity. Science 322: 590-594, 2008.

11. Yang YD, Cho H, Koo JY, et al: TMEM16A confers receptor-activated calcium-dependent chloride conductance. Nature 455: 1210-1215, 2008.

12. Schroeder BC, Cheng T, Jan YN and Jan LY: Expression cloning of TMEM16A as a calcium-activated chloride channel subunit. Cell 134: 1019-1029, 2008.

13. Rock JR, O'Neal WK, Gabriel SE, et al: Transmembrane protein 16A (TMEM16A) is a $\mathrm{Ca}^{2+}$-regulated $\mathrm{Cl}^{-}$secretory channel in mouse airways. J Biol Chem 284: 14875-14880, 2009.

14. Huang F, Rock JR, Harfe BD, et al: Studies on expression and function of the TMEM16A calcium-activated chloride channel. Proc Natl Acad Sci USA 106: 21413-21418, 2009.

15. Hwang SJ, Blair PJ, Britton FC, et al: Expression of anoctamin 1/TMEM16A by interstitial cells of Cajal is fundamental for slow wave activity in gastrointestinal muscles. J Physiol 587: 4887-4904, 2009.

16. Romanenko VG, Catalán MA, Brown DA, et al: Tmem16A encodes the $\mathrm{Ca}^{2+}$-activated $\mathrm{Cl}^{-}$channel in mouse submandibular salivary gland acinar cells. J Biol Chem 285: 12990-13001, 2010.

17. Elble RC and Pauli BU: Tumor suppression by a proapoptotic calcium-activated chloride channel in mammary epithelium. J Biol Chem 276: 40510-40517, 2001.

18. Spitzner M, Martins JR, Soria RB, et al: Eag1 and Bestrophin 1 are up-regulated in fast-growing colonic cancer cells. J Biol Chem 283: 7421-7428, 2008.

19. Katoh M and Katoh M: FLJ10261 gene, located within the CCND1-EMS1 locus on human chromosome 11q13, encodes the eight-transmembrane protein homologous to C12orf3, C11orf 25 and FLJ34272 gene products. Int J Oncol 22: 1375-1381, 2003.

20. Akervall JA, Jin Y, Wennerberg JP, et al: Chromosomal abnormalities involving 11q13 are associated with poor prognosis in patients with squamous cell carcinoma of the head and neck. Cancer 76: 853-859, 1995.

21. Schwab M: Amplification of oncogenes in human cancer cells. Bioessays 20: 473-479, 1998.

22. Carles A, Millon R, Cromer A, et al: Head and neck squamous cell carcinoma transcriptome analysis by comprehensive validated differential display. Oncogene 25: 1821-1831, 2006.

23. Espinosa I, Lee CH, Kim MK, et al: A novel monoclonal antibody against DOG1 is a sensitive and specific marker for gastrointestinal stromal tumors. Am J Surg Pathol 32: 210-218, 2008.

24. Kashyap MK, Marimuthu A, Kishore CJ, et al: Genomewide mRNA profiling of esophageal squamous cell carcinoma for identification of cancer biomarkers. Cancer Biol Ther 8: 36-46, 2009.

25. Yang H, Huang LY, Zeng DY, et al: Decrease of intracellular chloride concentration promotes endothelial cell inflammation by activating nuclear factor-kappaB pathway. Hypertension 60 : 1287-1293, 2012.

26. Chen J, Fok KL, Chen H, et al: Cryptorchidism-induced CFTR down-regulation results in disruption of testicular tight junctions through up-regulation of NF- $\mathrm{B} / \mathrm{COX}-2 / \mathrm{PGE} 2$. Hum Reprod 27: 2585-2597, 2012 
27. He G, Ma Y, Chou SY, et al: Role of CLIC4 in the host innate responses to bacterial lipopolysaccharide. Eur J Immunol 41: 1221-1230, 2011.

28. Sheridan GK, Pickering M, Twomey C, et al: NF-kappaB activity in distinct neural subtypes of the rat hippocampus: Influence of time and GABA antagonism in acute slice preparations. Learn Mem 14: 525-532, 2007.

29. Miller FJ, Jr, Filali M, Huss GJ, et al: Cytokine activation of nuclear factor kappa B in vascular smooth muscle cells requires signaling endosomes containing Nox1 and ClC-3. Circ Res 101: 663-671, 2007.

30. Laver T, Nozell S and Benveniste EN: The NF- $\kappa$ B signaling pathway in GBMs: implications for apoptotic and inflammatory responses and exploitation for therapy. In: CNS Cancer: Models, Markers, Prognostic Factors, Targets and Therapeutic Approaches. Van Meir EG (ed). Vol 1. Humana Press (Springer), New York, NY, pp1011-1036, 2009.

31. Gilmore TD: Introduction to NF-kappaB: players, pathways, perspectives. Oncogene 25: 6680-6684, 2006.

32. Perkins ND: The Rel/NF-kappa B family: friend and foe. Trends Biochem Sci 25: 434-440, 2000.

33. Perkins ND and Gilmore TD: Good cop, bad cop: the different faces of NF-kappaB. Cell Death Differ 13: 759-772, 2006.

34. Louis DN, Ohgaki H, Wiestler OD, et al: The 2007 WHO classification of tumours of the central nervous system. Acta Neuropathol 114: 97-109, 2007.

35. Livak KJ and Schmittgen TD: Analysis of relative gene expression data using real-time quantitative PCR and the 2(-Delta Delta C(T)) method. Methods 25: 402-408, 2001

36. Lee CH, Jeon YT, Kim SH and Song YS: NF-kappaB as a potential molecular target for cancer therapy. Biofactors 29: 19-35, 2007.

37. Sethi G, Sung B and Aggarwal BB: Nuclear factor-kappaB activation: from bench to bedside. Experiment Biol Med (Maywood) 233: 21-31, 2008

38. Van Waes C: Nuclear factor-kappaB in development, prevention, and therapy of cancer. Clin Cancer Res 13: 1076-1082, 2007.

39. Bauvois B: New facets of matrix metalloproteinases MMP-2 and MMP-9 as cell surface transducers: outside-in signaling and relationship to tumor progression. Biochim Biophys Acta 1825: 29-36, 2012.

40. Veeravalli KK and Rao JS: MMP-9 and uPAR regulated glioma cell migration. Cell Adh Migr 6: 509-512, 2012.

41. Kesanakurti D, Chetty C, Rajasekhar Maddirela D, Gujrati M and Rao JS: Functional cooperativity by direct interaction between PAK4 and MMP-2 in the regulation of anoikis resistance, migration and invasion in glioma. Cell Death Dis 3: e445, 2012.
42. Habela CW, Ernest NJ, Swindall AF and Sontheimer H Chloride accumulation drives volume dynamics underlying cell proliferation and migration. J Neurophysiol 101: 750-757, 2009.

43. Habela CW, Olsen ML and Sontheimer H: ClC3 is a critical regulator of the cell cycle in normal and malignant glial cells. J Neurosci 28: 9205-9217, 2008.

44. Kunzelmann K, Kongsuphol P, Aldehni F, et al: Bestrophin and TMEM16-Ca(2+) activated $\mathrm{Cl}(-)$ channels with different functions. Cell Calcium 46: 233-241, 2009.

45. Ayoub C, Wasylyk C, Li Y, et al: ANO1 amplification and expression in HNSCC with a high propensity for future distant metastasis and its functions in HNSCC cell lines. Br J Cancer 103: 715-726, 2010

46. Duvvuri U, Shiwarski DJ, Xiao D, et al: TMEM16A induces MAPK and contributes directly to tumorigenesis and cancer progression. Cancer Res 72: 3270-3281, 2012.

47. Simon S, Grabellus F, Ferrera L, et al: DOG1 regulates growth and IGFBP5 in gastrointestinal stromal tumors. Cancer Res 73: 3661-3670, 2013.

48. Mazzone A, Eisenman ST, Strege PR, et al: Inhibition of cell proliferation by a selective inhibitor of the $\mathrm{Ca}(2+)$-activated $\mathrm{Cl}(-)$ channel, Ano1. Biochem Biophys Res Commun 427: 248-253, 2012.

49. Liu W, Lu M, Liu B, Huang Y and Wang K: Inhibition of $\mathrm{Ca}(2+)$-activated $\mathrm{Cl}(-)$ channel ANO1/TMEM16A expression suppresses tumor growth and invasiveness in human prostate carcinoma. Cancer Lett 326: 41-51, 2012.

50. Abdullah JM, Ahmad F, Ahmad KA, et al: Molecular genetic analysis of BAX and Cyclin D1 genes in patients with malignant glioma. Neurol Res 29: 239-242, 2007.

51. Arato-Ohshima T and Sawa H: Over-expression of cyclin D1 induces glioma invasion by increasing matrix metalloproteinase activity and cell motility. Int J Cancer 83: 387-392, 1999.

52. Zhang X, Zhao M, Huang AY, et al: The effect of cyclin D expression on cell proliferation in human gliomas. J Clin Neurosci 12: 166-168, 2005.

53. Liao DJ, Thakur A, Wu J, Biliran H and Sarkar FH: Perspectives on c-Myc, cyclin D1, and their interaction in cancer formation, progression, and response to chemotherapy. Crit Rev Oncogen 13: 93-158, 2007

54. Robson S, Pelengaris S and Khan M: c-Myc and downstream targets in the pathogenesis and treatment of cancer. Recent Pat Anticancer Drug Discov 1: 305-326, 2006. 